\title{
Cooperative Transmission for Wireless Relay Networks Using Limited Feedback
}

\author{
Javier M. Paredes, Babak H. Khalaj, and Alex B. Gershman
}

\begin{abstract}
To achieve the available performance gains in half-duplex wireless relay networks, several cooperative schemes have been earlier proposed using either distributed space-time coding or distributed beamforming for the transmitter without and with channel state information (CSI), respectively. However, these schemes typically have rather high implementation and/or decoding complexities, especially when the number of relays is high. In this paper, we propose a simple low-rate feedback-based approach to achieve maximum diversity with a low decoding and implementation complexity. To further improve the performance of the proposed scheme, the knowledge of the second-order channel statistics is exploited to design long-term power loading through maximizing the receiver signal-to-noise ratio (SNR) with appropriate constraints. This maximization problem is approximated by a convex feasibility problem whose solution is shown to be close to the optimal one in terms of the error probability. Subsequently, to provide robustness against feedback errors and further decrease the feedback rate, an extended version of the distributed Alamouti code is proposed. It is also shown that our scheme can be generalized to the differential transmission case, where it can be applied to wireless relay networks with no CSI available at the receiver.
\end{abstract}

\section{Index Terms}

Cooperative communications, distributed space-time coding, limited feedback, wireless relay networks

J. M. Paredes and A. B. Gershman are with the Communication Systems Group, Darmstadt University of Technology, Merckstr. 25, D-64283 Darmstadt, Germany; Phone: +49-6151-164970; Fax: +49-6151-166095; e-mails: jparedes, gershmanent.tu-darmstadt.de. B. H. Khalaj is with the Department of Electrical Eng., Sharif University of Technology, Tehran, Iran; e-mail: khalajesharif.ir. The work of B. H. Khalaj was supported by a Research Fellowship from the Alexander von Humboldt Foundation. The work of A. B. Gershman was supported in part by the European Research Council (ERC) under Advanced Investigator Grant program and the German Research Foundation (DFG) under Grant GE 1881/4-1. 


\section{INTRODUCTION}

The performance of wireless communication systems can be severely affected by channel fading. To combat fading, multi-antenna systems are commonly used as in such systems, the existence of independent paths between the transmitter and receiver can be used to achieve a higher degree of diversity than in single-antenna systems [1]-[3]. However, restrictions in size and hardware costs can make the use of multi-antenna systems impractical in wireless networks. Fortunately, similar independent paths are also available in wireless networks with multiple single-antenna nodes, where some nodes are used as relays that help to convey the information through the network. Using such relays between the transmitter and receiver nodes offers the so-called cooperative diversity and, hence, can be a good alternative to using multiple antennas at the transmitter and/or receiver. Several cooperation methods between network nodes have been proposed based on different relaying strategies; see [4]-[10] and references therein.

Among different relaying approaches, techniques using the amplify-and-forward relaying strategy are of especial practical interest because they do not require any signal processing (such as decoding or compression) at the relays.

The use of space-time codes (originally developed for multi-antenna systems [11], [12]) in a distributed fashion has been proposed for relay networks in [4] and [13] using the amplify-and-forward approach. In this cooperative strategy, the source terminal first transmits the information symbols to the relays. Then, the relays encode their received signals and their conjugates in a linear fashion and transmit them to the destination node. This can be viewed as distributed space-time coding (DSTC). The DSTC techniques only require the knowledge of the received signal powers at the relays and can achieve the maximum diversity available in the network. In [14], orthogonal space-time block codes (OSTBCs) [12], [15] and quasi-orthogonal STBCs (QOSTBCs) [16], [17] have been used along with the DSTC strategy of [13]. Both these DSTC approaches have been shown to offer maximum diversity, optimal diversity products, low maximum likelihood (ML) decoding complexity, linear encoding of the information symbols, and robustness against relay failures. Unfortunately, for more than two relays, the maximum rate of OSTBCs reduces [18], the decoding delay increases, and the linear ML decoding complexity is no longer achievable [14]. Furthermore, QOSTBCs are only applicable to particular cases with certain numbers of relays. In addition, their decoding complexity is higher than that of OSTBCs.

In [19], four-group decodable DSTC 1 for any number of relays are proposed. Although this approach reduces the decoding complexity as compared to the full ML decoder, its complexity is still rather high,

\footnotetext{
${ }^{1}$ For these codes, it is possible to split the maximum likelihood decoding problem into four independent subproblems.
} 
especially in the case of more than four relays. To recover the simple symbol-by-symbol ML decoding property of the distributed OSTBCs for more than two relays, the use of the source-to-relay CSI at the relays has been proposed [14], [20]. However, as shown in [14], this does not improve the resulting diversity or coding gains.

Another promising approach to amplify-and-forward relaying in wireless networks is distributed beamforming; see [21]-[26] and references therein. As most of distributed beamforming techniques require the full knowledge of the instantaneous CSI for both the source-to-relay and relay-to-destination links and, moreover, require a feedback link between the destination and relays, the complexities of these techniques are rather high. To decrease the distributed beamforming complexity, the use of quantized feedback for selecting beamforming weights from a codebook has been proposed in [27]. However, the codebook design requires a costly numerical optimization and the resulting codebook needs to be transmitted to each relay every time when the channel statistics or the transmitted powers change.

In this paper (see also [28] and [29]), we consider a wireless network where each relay only needs to know its average received signal power (which is a common assumption for DSTCs) and further assume that one-bit feedback per relay is available for every channel realization. The proposed scheme is based on the ideas of partial phase combining (PPC) [30], [31] and the group coherent codes (GCCs) [32] originally introduced for traditional multiple-antenna systems. It will be shown by means of an approximate symbol error rate (SER) analysis that such a low-rate feedback is sufficient to achieve maximum diversity with an additional coding (power) gain. Furthermore, the proposed scheme will be shown to enjoy linear decoding complexity and minimum decoding delay for any number of relays. Although the best possible choice for the feedback bits has to be found by a full search, we provide two much simpler methods to judiciously choose these bits.

It should be noted that several techniques related to the proposed scheme have been developed in [33][35] in the context of sensor networks. In these papers, randomly generated relay beamformer phases are iteratively selected based on a low-rate feedback. In particular, in [35] the application of binary signaling to the approach of [33], [34] has been considered. The scheme of [33]-[35] requires multiple iterations to converge, and the number of such iterations is in average comparable to or larger than the number of sensor nodes. In contrast to the approaches of [33]-[35], the proposed scheme will use a fixed (substantially lower) number of feedback bits without any need for multiple iterations.

Since the quality of channel links can vary for different relays, we propose to use second-order channel statistics to find proper "long-term" power loading coefficients for each relay. From the performance viewpoint, these coefficients should be designed by minimizing the error probability as it was proposed 
in [36] for a two-relay network using the distributed Alamouti code. However, the approach of [36] does not provide any extension to the case of more than two relays. As an alternative, we propose to use the general idea of [24] to obtain the power loading coefficients by maximizing the average SNR subject to individual power constraints. However, in contrast to [24], the loss in diversity is avoided by a proper choice of the instantaneous feedback bits and by using appropriate constraints on these coefficients. It is shown that using semi-definite relaxation (SDR), the resulting SNR maximization problem can be turned into a convex feasibility problem which can be efficiently solved using interior point methods. Simulations show that the resulting solution performs very close to the direct (computationally prohibitive) approach that minimizes the Chernoff bound on the error probability using brute force optimization.

Using an extended version of the distributed Alamouti code, we further refine the proposed technique to reduce the amount of feedback without affecting the benefits of linear decoding complexity and maximum diversity. In addition, the use of such an extended distributed Alamouti code is shown to provide extra robustness to erroneous feedback.

Finally, an extension of the proposed scheme for non-coherent receivers using differential transmission is developed. It is demonstrated that the proposed non-coherent scheme enjoys the same advantages in performance, decoding complexity and delay as its coherent counterpart.

The remainder of this paper is organized as follows. In Section 【 the system model is developed. Section $\amalg$ presents the proposed scheme. Its further refinement using the extended distributed Alamouti code is discussed in Section [V] The differential transmission extension of the proposed techniques is developed in Section $\nabla$. Computer simulations are presented in Section VI and conclusions are drawn in Section VII

\section{SySTEM MODEL}

Let us consider a half-duplex wireless relay network with $R+2$ nodes where each node has a single antenna that can transmit or receive signals. Among these $R+2$ nodes, one is the transmitter, one is the receiver, and the remaining $R$ nodes are the relays. It is assumed that the direct link between the transmitter and the receiver can not be established and that the relay channels are statistically independent. We consider the quasi-static flat fading channel case with the block length $T$, and denote the channel coefficient between the transmitter and the $i$ th relay by $f_{i}$. Correspondingly, the channel coefficient between the $i$ th relay and the receiver is denoted by $g_{i}$. We assume that $f_{i}$ and $g_{i}$ are independent random variables with the probability density functions (pdf's) $\mathcal{C N}\left(\mu_{f_{i}}, \sigma_{f_{i}}^{2}\right)$ and $\mathcal{C N}\left(\mu_{g_{i}}, \sigma_{g_{i}}^{2}\right)$, respectively, where $\mathcal{C N}(\cdot, \cdot)$ denotes the complex Gaussian pdf. 
We assume that the transmitter does not have any CSI. However, we consider a limited feedback link between the receiver and each relay. This feedback link is used to transmit one bit for every channel realization and can be also used to transmit long-term power loading coefficients (one per relay) every time the channel means or variances change significantly. The receiver may or may not enjoy full CSI, depending on the transmission mode (coherent or non-coherent) and the system is synchronized at the symbol level.

At the transmitter side, $T$ symbols $\mathbf{s}=\left[s_{1}, \ldots, s_{T}\right]^{T}$ are drawn from an $M$-point constellation according to the information bits to be sent. Here, $(\cdot)^{T}$ denotes the transpose. The signal $\mathbf{s}$ is normalized as $\mathrm{E}\left\{\mathbf{s}^{H} \mathbf{s}\right\}=1$, where $(\cdot)^{H}$ and $\mathrm{E}\{\cdot\}$ denote the Hermitian transpose and the statistical expectation, respectively. The transmission is carried out in two steps. In the first step, the transmitter sends $\sqrt{P_{0} T} \mathbf{s}$ from time 1 to $T$, where $P_{0}$ is its average transmitted power. The received signal at the $i$ th relay is given by

$$
\mathbf{r}_{i}=\sqrt{P_{0} T} f_{i} \mathbf{s}+\mathbf{v}_{i}
$$

where $\mathbf{v}_{i}$ is the noise vector at the $i$ th relay. In the second step, the $i$ th relay sends the signal $\mathbf{d}_{i}$ to the receiver from time $T+1$ to $2 T$. At the receiver, we have

$$
\mathbf{x}=\sum_{i=1}^{R} g_{i} \mathbf{d}_{i}+\mathbf{n}
$$

where $\mathbf{x}=\left[x_{1}, \ldots, x_{T}\right]^{T}$ is the received signal and $\mathbf{n}$ is the receiver noise vector. We assume that the entries of the noise vectors $\mathbf{v}_{i}$ and $\mathbf{n}$ are i.i.d. random variables with the pdf $\mathcal{C N}(0,1)$, that is, both these noises have variance $\sigma^{2}=1$.

The transmitted signal $\mathbf{d}_{i}$ at each relay is assumed to be a linear function of its received signal and its conjugate [14], that is,

$$
\begin{aligned}
\mathbf{d}_{i} & =\sqrt{\frac{P_{i}}{m_{f_{i}} P_{0}+1}} b_{i} \theta_{i}\left(\mathbf{A}_{i} \mathbf{r}_{i}+\mathbf{B}_{i} \mathbf{r}_{i}^{*}\right) \\
& =\sqrt{\frac{P_{0} P_{i} T}{m_{f_{i}} P_{0}+1}} b_{i} \theta_{i}\left(f_{i} \mathbf{A}_{i} \mathbf{s}+f_{i}^{*} \mathbf{B}_{i} \mathbf{s}^{*}\right)+\sqrt{\frac{P_{i}}{m_{f_{i}} P_{0}+1}} b_{i} \theta_{i}\left(\mathbf{A}_{i} \mathbf{v}_{i}+\mathbf{B}_{i} \mathbf{v}_{i}^{*}\right)
\end{aligned}
$$

where $m_{f_{i}} \triangleq \mathrm{E}\left\{\left|f_{i}\right|^{2}\right\}=\left|\mu_{f_{i}}\right|^{2}+\sigma_{f_{i}}^{2}, b_{i} \in\{-1,1\}$ is a coefficient selected based on the value of the one-bit feedback, $\theta_{i}\left(0 \leq \theta_{i} \leq 1\right)$ is a real-valued long-term power loading coefficient that is adjusted according to the channel statistics (as it will be explained in Section $\amalg$ II-B), $P_{i}$ is the maximum average power available at the $i$ th relay (while the actual transmitted power is $\left.P_{i} \theta_{i}^{2} \leq P_{i}\right),(\cdot)^{*}$ denotes the complex conjugate and the $T \times T$ matrices $\mathbf{A}_{i}$ and $\mathbf{B}_{i}$ are assumed to be either $\mathbf{A}_{i}=\mathbf{O}$ with $\mathbf{B}_{i}$ being unitary, or $\mathbf{B}_{i}=\mathbf{O}$ with $\mathbf{A}_{i}$ being unitary. Here, $\mathbf{O}$ is the $T \times T$ matrix of zeros. This assumption implies 
that the statistics of the noise remains unchanged and that the transmitted signal at each relay depends either on its received signal or on the complex conjugate of this signal.

Using this model, let us introduce the following notations:

$$
\begin{aligned}
& \text { If } \mathbf{B}_{i}=\mathbf{O} \text { then } \tilde{\mathbf{A}}_{i}=\mathbf{A}_{i}, \tilde{f}_{i}=f_{i}, \tilde{\mathbf{v}}=\mathbf{v}_{i}, \tilde{\mathbf{s}}_{i}=\mathbf{s} \\
& \text { If } \mathbf{A}_{i}=\mathbf{O} \text { then } \tilde{\mathbf{A}}_{i}=\mathbf{B}_{i}, \tilde{f}_{i}=f_{i}^{*}, \tilde{\mathbf{v}}=\mathbf{v}_{i}^{*}, \tilde{\mathbf{s}}_{i}=\mathbf{s}^{*}
\end{aligned}
$$

Taking into account (3)-(5), the received signal model (2) can be written as

$$
\mathbf{x}=\mathbf{S}(\mathbf{p} \odot \mathbf{h})+\mathbf{w}
$$

where

$$
\mathbf{S} \triangleq\left[\tilde{\mathbf{A}}_{1} \tilde{\mathbf{s}}_{1}, \ldots, \tilde{\mathbf{A}}_{R} \tilde{\mathbf{s}}_{R}\right]
$$

is the distributed space-time code matrix,

$$
\mathbf{h}=\left[h_{1}, \ldots, h_{R}\right]^{T} \triangleq\left[\tilde{f}_{1} g_{1}, \ldots, \tilde{f}_{R} g_{R}\right]^{T}
$$

is the equivalent channel vector,

$$
\mathbf{w}=\left[w_{1}, \ldots, w_{T}\right]^{T} \triangleq \sum_{i=1}^{R} \sqrt{\frac{P_{i}}{m_{f_{i}} P_{0}+1}} b_{i} \theta_{i} g_{i} \tilde{\mathbf{A}}_{i} \tilde{\mathbf{v}}_{i}+\mathbf{n}
$$

is the equivalent noise vector,

$$
\mathbf{p} \triangleq\left[\sqrt{\frac{P_{0} P_{1} T}{m_{f_{i}} P_{0}+1}} b_{1} \theta_{1}, \ldots, \sqrt{\frac{P_{0} P_{R} T}{m_{f_{R}} P_{0}+1}} b_{R} \theta_{R}\right]^{T}
$$

and $\odot$ denotes the Schur-Hadamard (element-wise) matrix product.

\section{The Proposed Cooperative Transmission Scheme}

In this section, we address the problem of selecting the coefficients $b_{i}(i=1, \ldots, R)$ and the long-term power loading coefficients $\theta_{i}(i=1, \ldots, R)$. We assume that the value of $\mathbf{p} \odot \mathbf{h}$ is known at the receiver and there is a perfect (error-free) low-rate feedback link between the receiver and the relays. We will first introduce the transmission strategy based on one-bit feedback per relay to choose the coefficients $b_{i}$ for every channel realization. It will be shown that this transmission scheme achieves maximum diversity. Subsequently, a further improvement of this scheme will be considered using an additional long-term real-valued power loading coefficient to feed back from the receiver to each relay. These coefficients will be computed using second-order channel statistics.

For the sake of simplicity, in this section we assume that $T=1$. Hence, matrices $\mathbf{A}_{i}$ and $\mathbf{B}_{i}$ become scalars and it is assumed that $\mathbf{A}_{i}=1$ and $\mathbf{B}_{i}=0$. Correspondingly, $\mathbf{x}, \mathbf{v}_{i}, \mathbf{n}, \mathbf{w}$ and $\mathbf{s}$ become scalars 
as well. A more general case when $T>1$ (and when $\mathbf{A}_{i}$ and $\mathbf{B}_{i}$ are matrices rather than scalars) will be considered in Section IV

\section{A. Using One-Bit Feedback Per Relay}

As in the case of one-bit feedback the long-term power loading is not taken into account, all the relays transmit with the maximum power $P_{i}$ (i.e., $\theta_{i}=1$ for $i=1, \ldots, R$ ). In this particular case, the received signal model (6) reduces to

$$
x=\mathbf{1}_{R}^{T}(\mathbf{p} \odot \mathbf{h}) s+w
$$

where $1_{R}$ is the $R \times 1$ column vector of ones. For the sake of simplicity, the sub-indices in all scalar values are hereafter omitted.

Using (7) and (9), the noise power can be expressed as

$$
P_{w}=\mathrm{E}\left\{|n|^{2}\right\}+\sum_{i=1}^{R} \frac{P_{i}}{m_{f_{i}} P_{0}+1} \mathrm{E}\left\{\left|v_{i}\right|^{2}\right\} b_{i}^{2}\left|g_{i}\right|^{2}=1+\sum_{i=1}^{R} \frac{P_{i}}{m_{f_{i}} P_{0}+1}\left|g_{i}\right|^{2} .
$$

From (10) it is clear that the choice of $b_{i}$ does not affect the noise power. Using (9), the signal power can be obtained as

$$
\begin{aligned}
P_{s} & =\left|\mathbf{1}_{R}^{T}(\mathbf{p} \odot \mathbf{h})\right|^{2} \mathrm{E}\left\{|s|^{2}\right\} \\
& =\left|\sum_{i=1}^{R} \sqrt{\frac{P_{0} P_{i}}{m_{f_{i}} P_{0}+1}} b_{i} f_{i} g_{i}\right|^{2}=\underbrace{\sum_{i=1}^{R} \rho_{i, i}\left|f_{i} g_{i}\right|^{2}}_{\gamma}+\underbrace{\sum_{\substack{i, j=1 \\
i \neq j}}^{R} \rho_{i, j} b_{i} b_{j} \operatorname{Re}\left\{f_{i} g_{i} f_{j}^{*} g_{j}^{*}\right\}}_{\beta}
\end{aligned}
$$

where

$$
\rho_{i, j} \triangleq \sqrt{\frac{T P_{0} P_{i}}{m_{f_{i}} P_{0}+1}} \sqrt{\frac{T P_{0} P_{j}}{m_{f_{j}} P_{0}+1}}
$$

for $i, j=1, \ldots, R$, and $\operatorname{Re}\{\cdot\}$ denotes the real part operation. In general, $\beta$ can take negative values. Clearly, such negative values of $\beta$ will reduce the received SNR and affect the achieved diversity. Our key idea here is to use the coefficients $b_{i}$ to ensure that $\beta$ is always non-negative. It can be proved using the same approach as presented in [32] that using values of $b_{i} \in\{-1,1\}$ is sufficient to guarantee $\beta \geq 0$. This results in a scheme with the diversity order proportional to $R$, as stated in the following proposition.

Proposition 1: If $\beta \geq 0$, then the average symbol error probability ( $\overline{\mathrm{SER}}$ ) for (9) can be upper bounded by

$$
\overline{\mathrm{SER}} \leq \kappa P^{-R\left(1-\frac{\log \log P}{\log P}\right)}
$$

for large $R$ and large $P$, where $P$ is the total power in the network and $\kappa$ is a constant. 
Proof: See Appendix.

It follows from Proposition 1 that the achievable diversity order of the proposed scheme is $R$.

Since positive values of $\beta$ will provide an additional signal power gain, the optimal values of $b_{i}$ $(i=1, \ldots, R)$ can be obtained through maximizing $\beta$. This is an integer maximization problem that requires a full search over all possible values of $b_{i}$. Clearly, if the number of relays is large, then such a full search procedure can be impractical. To reduce the complexity, we propose a near-optimal solution based on SDR, that we denote hereafter as Algorithm 1.

Note that, according to (11), the choice of $b_{i}$ does not affect the value of $\gamma$. Therefore, to maximize $P_{s}$, it is sufficient to maximize $\beta$ in (11). Let us express $P_{s}$ in a more convenient form by extending the notation for $\rho_{i, j}$ in (12) with

$$
\rho_{i, 0} \triangleq \sqrt{\frac{P_{i} P_{0} T}{m_{f_{i}} P_{0}+1}}
$$

and denoting

$$
\overline{\mathbf{h}} \triangleq\left[\rho_{1,0} f_{1} g_{1}, \ldots, \rho_{R, 0} f_{R} g_{R}\right]^{T}
$$

Using (14), the signal power (11) can be expressed as

$$
P_{s}=\left|\overline{\mathbf{h}}^{H} \mathbf{b}\right|^{2}
$$

where $\mathbf{b} \triangleq\left[b_{1}, \ldots, b_{R}\right]^{T}$. Defining $\overline{\mathbf{Q}} \triangleq \overline{\mathbf{h}} \overline{\mathbf{h}}^{H}$, we can write the optimization problem as

$$
\max _{\mathbf{b} \in\{-1,1\}^{R}} \mathbf{b}^{T} \overline{\mathbf{Q}} \mathbf{b} .
$$

As $\mathbf{b}^{T} \overline{\mathbf{Q}} \mathbf{b}=\operatorname{tr}\left(\mathbf{b} \mathbf{b}^{T} \overline{\mathbf{Q}}\right)$, the optimization problem in (15) can be rewritten as

$$
\max _{\mathbf{B}} \operatorname{tr}(\mathbf{B} \overline{\mathbf{Q}}) \quad \text { s.t. } \quad \operatorname{rank}\{\mathbf{B}\}=1, \quad \mathbf{B} \succeq 0, \quad[\mathbf{B}]_{i i}=1, \quad i=1, \ldots, R
$$

where $\mathbf{B} \triangleq \mathbf{b b}^{T}, \mathbf{b} \in \mathbb{R}^{R}, \operatorname{tr}(\cdot)$ stands for the trace of a matrix, $\mathbb{R}$ denotes the set of real numbers, and $[\mathbf{B}]_{i i}$ denotes the $i$ th diagonal element of B. Problems similar to (16) arise in the context of ML detection. Solutions close to the optimal one can be efficiently found using the SDR approach [37], whose essence is to omit the rank-one constraint $\operatorname{rank}\{\mathbf{B}\}=1$ in $(16)$ and, therefore, approximate the latter non-convex problem by a convex problem

$$
\max _{\mathbf{B}} \operatorname{tr}(\mathbf{B} \overline{\mathbf{Q}}) \quad \text { s.t. } \quad \mathbf{B} \succeq 0,[\mathbf{B}]_{i i}=1, i=1, \ldots, R .
$$

Note that this problem can be efficiently solved using interior point techniques [37]-[39]. Generally, the resulting solution for $\mathbf{B}$ is not guaranteed to be rank-one. If it is rank-one, then its principal eigenvector 
is the optimal solution to (15). Otherwise, a proper approximate solution for $\boldsymbol{\theta}$ can be recovered from $\mathbf{B}$ using randomization techniques, see [37] and [39] for more detail.

Thus, our SDR-based approach can be summarized as follows.

\section{Algorithm 1}

1. At the receiver, find the solution to (17) using the approach of [37].

2. Send the so-obtained $b_{i}$ from the receiver to the $i$ th relay node for each $i=1, \ldots, R$ using one-bit per relay feedback.

As it will be shown throughout our simulations, the use of the SDR approach results in a performance that is very close to that of the full search-based approach. The complexity of the SDR approach is much lower than that of the full search; see [37] for details.

To further reduce the complexity, let us discuss a simpler algorithm to obtain acceptable values of $b_{i}$ that can be formulated using the general idea of [32]. The essence of this algorithm is to use a greedy selection of the values of $b_{i}$ in a consecutive way. This algorithm can be summarized as the following sequence of steps:

\section{Algorithm 2}

1. Set $b_{1}=1$ and $\tau_{1}=h_{1}$.

2. For $i=2, \ldots, R$, compute

$$
b_{i}=\operatorname{sign}\left(\operatorname{Re}\left\{h_{i}^{*} \tau_{i-1}\right\}\right), \quad \tau_{i}=\tau_{i-1}+b_{i} h_{i}
$$

where $\operatorname{sign}(\cdot)$ is the sign function.

3. Send the so-obtained $b_{i}$ from the receiver to the $i$ th relay node for each $i=1, \ldots, R$ using one-bit per relay feedback.

Note that Algorithm 2 does not result in the optimal values of $b_{i}, i=1, \ldots, R$. However, Algorithm 2 is computationally much simpler than Algorithm 1. Hence, these two alternative techniques are expected to provide different performance-to-complexity tradeoffs.

\section{B. Choosing Long-Term Power Loading}

So far, we have not considered the use of power loading, $\theta_{i}$, for each relay. In practical scenarios, relays are distributed randomly in an area between the transmitter and the receiver. As a result, the power loss characteristics in the source-to-relay and relay-to-destination links are different for each relay. Furthermore, different relays may have different transmitted power constraints. Therefore, in such 
scenarios, some power loading strategy should be employed to take into account such differences in channel quality and/or constraints on the relay transmitted power.

From the performance viewpoint, the optimal power loading should be designed by minimizing the error probability as proposed in [36] for a two-relay network using the distributed Alamouti code. However, the approach of [36] does not provide any extension to the case of more than two relays. As an alternative to the error probability criterion, we propose to use the general idea of [24] to obtain the power loading coefficients by maximizing the average SNR subject to individual power constraints.

In what follows, the maximization of the average received SNR is used as a criterion to design the power loading coefficients $\theta_{i}$. Note that a related strategy to choose the beamforming weights was also used in [24]. However, we will show that in contrast to [24], full diversity can be achieved in our case by using the optimal feedback values $b_{i}$ along with the coefficients $\theta_{i}$.

First, let us evaluate the average signal power, that is

$$
\mathrm{E}\left\{P_{s}\right\}=\mathrm{E}\{\tilde{\gamma}\}+\mathrm{E}\{\tilde{\beta}\}
$$

where

$$
\begin{aligned}
& \tilde{\gamma} \triangleq \sum_{i=1}^{R} \rho_{i, i} \theta_{i}^{2}\left|f_{i} g_{i}\right|^{2} \\
& \tilde{\beta} \triangleq \sum_{\substack{i, j=1 \\
i \neq j}}^{R} \rho_{i, j} \theta_{i} \theta_{j} b_{i} b_{j} \operatorname{Re}\left\{f_{i} g_{i} f_{j}^{*} g_{j}^{*}\right\} .
\end{aligned}
$$

Note that in (18), the analytical evaluation of $\mathrm{E}\{\tilde{\beta}\}$ is very difficult due to the dependence of $b_{i}$ $(i=1, \ldots, R)$ on the instantaneous channel values. Therefore, using (19)-(20) and assuming that the optimal $b_{i}(i=1, \ldots, R)$ are selected, we propose to approximate (18) as

$$
\mathrm{E}\left\{P_{s}\right\} \approx \sum_{i, j=1}^{R} \rho_{i, j} \theta_{i} \theta_{j}\left|\operatorname{Re}\left\{\mathrm{E}\left\{f_{i} g_{i} f_{j}^{*} g_{j}^{*}\right\}\right\}\right| .
$$

The quality of this approximation is illustrated in Fig. 1 where the exact value of $\mathrm{E}\left\{P_{s}\right\}$ and its approximation (21) are plotted versus $P$ normalized by the noise variance $\sigma^{2}$. In this figure, it is assumed that $R=4$ and $\theta_{i}=1(i=1, \ldots, R)$. All the channels are assumed to be complex circular Gaussian random variables with zero-mean and unit variance.

Another important question when using this approximation is how close the values of achieved average SNR obtained from the approximation in (21) and from the exact value of $\mathrm{E}\left\{P_{s}\right\}$ are. This question was investigated by means of extensive Monte-Carlo simulations that, for the sake of brevity, are not presented in all detail. These simulations have involved different channel scenarios, randomly generated 
channel coefficients for each particular scenario, and different numbers of relays lying in the interval $R=2, \ldots, 7$. The optimal coefficients $\theta_{i}$ have been obtained by brute force optimization of $\mathrm{E}\left\{P_{s}\right\}$ and their approximate values have been found by optimizing (21). Then, the achieved average SNRs were compared for two so-obtained sets of optimized power loading coefficients.

The results of this comparison have verified that the difference between the exact optimal SNR (computed numerically via brute force optimization of $\left.\mathrm{E}\left\{P_{s}\right\}\right)$ and its approximation computed via (21) is, in average, less than $3 \%$. This implies that the approximation (21) is worth using for maximizing the average SNR by power loading.

Using the statistical independence of all source-to-relay and relay-to-destination channels, we can now estimate the expected value in (21) as

$$
\mathrm{E}\left\{f_{i} g_{i} f_{j}^{*} g_{j}^{*}\right\}=\mathrm{E}\left\{f_{i} f_{j}^{*}\right\} \mathrm{E}\left\{g_{i} g_{j}^{*}\right\}=\left(\mu_{f_{i}} \mu_{f_{j}}^{*}+\delta_{i j} \sigma_{f_{i}}^{2}\right)\left(\mu_{g_{i}} \mu_{g_{j}}^{*}+\delta_{i j} \sigma_{g_{i}}^{2}\right)
$$

where $\delta_{i j}$ is the Kronecker delta.

Let us define the real-valued matrix $\mathbf{Q}$ with the $(i, j)$ entry as

$$
[\mathbf{Q}]_{i, j}=\rho_{i, j}\left|\operatorname{Re}\left\{\left(\mu_{f_{i}} \mu_{f_{j}}^{*}+\delta_{i j} \sigma_{f_{i}}^{2}\right)\left(\mu_{g_{i}} \mu_{g_{j}}^{*}+\delta_{i j} \sigma_{g_{i}}^{2}\right)\right\}\right|
$$

for $i, j=1, \ldots, R$. Using (22) and (23), equation (21) can be written as

$$
\mathrm{E}\left\{P_{s}\right\} \approx \boldsymbol{\theta}^{T} \mathbf{Q} \boldsymbol{\theta}
$$

where $\boldsymbol{\theta} \triangleq\left[\theta_{1}, \ldots, \theta_{R}\right]^{T}$. Using the fact that the noise waveforms and the channel coefficients are statistically independent, the noise power can be expressed as

$$
\mathrm{E}\left\{P_{w}\right\}=\mathrm{E}\left\{|n|^{2}\right\}+\sum_{i=1}^{R} \frac{\theta_{i}^{2} P_{i}}{m_{f_{i}} P_{0}+1} \mathrm{E}\left\{\left|v_{i}\right|^{2}\right\} \mathrm{E}\left\{\left|g_{i}\right|^{2}\right\}=1+\sum_{i=1}^{R} \frac{\theta_{i}^{2} P_{i} m_{g_{i}}}{m_{f_{i}} P_{0}+1}
$$

and further rewritten as

$$
\mathrm{E}\left\{P_{w}\right\}=\boldsymbol{\theta}^{T} \mathbf{W} \boldsymbol{\theta}+1
$$

where

$$
\mathbf{W} \triangleq \operatorname{diag}\left(\frac{m_{g_{1}} P_{1}}{m_{f_{1}} P_{0}+1}, \ldots, \frac{m_{g_{R}} P_{R}}{m_{f_{R}} P_{0}+1}\right)
$$

and $\operatorname{diag}(\cdot)$ denotes a diagonal matrix. Using (24) and (25), the maximization of the receiver SNR over $\boldsymbol{\theta}$ can be approximately written as

$$
\max _{\boldsymbol{\theta}} \frac{\boldsymbol{\theta}^{T} \mathbf{Q} \boldsymbol{\theta}}{\boldsymbol{\theta}^{T} \mathbf{W} \boldsymbol{\theta}+1} \quad \text { s.t. } \quad \theta_{i}^{2} \leq 1, \quad i=1, \ldots, R
$$

where instead of the signal power we use its approximation given by (21). 
If the aggregate power constraint $\left(\boldsymbol{\theta}^{T} \boldsymbol{\theta}=R\right)$ is used instead of the individual relay power constraints in (26), the resulting problem becomes

$$
\max _{\boldsymbol{\theta}} \frac{\boldsymbol{\theta}^{T} \mathbf{Q} \boldsymbol{\theta}}{\left.\boldsymbol{\theta}^{T}\left(\mathbf{W}+(1 / R) \mathbf{I}_{R}\right)\right) \boldsymbol{\theta}} \quad \text { s.t. } \quad \boldsymbol{\theta}^{T} \boldsymbol{\theta}=R .
$$

Solving (27) amounts to the unconstrained optimization of the objective function in 27) (that boils down to solving a generalized eigenvector problem) followed by rescaling the so-obtained vector $\boldsymbol{\theta}$ to satisfy the constraint $\boldsymbol{\theta}^{T} \boldsymbol{\theta}=R$.

In what follows, we consider a more practical case of individual power constraints rather than the aggregate power constraint.

As mentioned above, the design of power loading coefficients by maximizing the average SNR does not take into account the diversity aspect of the problem. In fact, maximizing the average SNR can result in a solution with a poor performance in terms of the error probability. This can particularly be the case if some of the resulting values of $\theta_{i}$ are small, so that the diversity order suffers. Indeed, if $\theta_{i}$ is close to zero at the $i$ th relay, then this is equivalent to switching off the $i$ th relay for all the transmissions within the time interval where the current value of $\theta_{i}$ is used. According to Proposition 1, this will reduce the diversity order.

To prevent such a loss in diversity, an additional constraint $\theta_{i}^{2} \geq \bar{\theta}_{i}^{2}$ can be used where $\bar{\theta}_{i}^{2}$ is a preselected minimum power loading value that establishes a tradeoff between the diversity and power loading performance. If $\bar{\theta}_{i}$ is chosen too large, then the interval for $\theta_{i}$ will be smaller, and this may prevent the scheme from achieving any significant improvement in the performance due to power loading. Reversely, if $\bar{\theta}_{i}$ is chosen too small, a substantial diversity loss can occur.

Defining $\Theta \triangleq \boldsymbol{\theta} \boldsymbol{\theta}^{T}$, we can rewrite (26) as

$$
\begin{gathered}
\max _{\boldsymbol{\Theta}} \frac{\operatorname{tr}(\mathbf{Q} \boldsymbol{\Theta})}{\operatorname{tr}(\mathbf{W} \boldsymbol{\Theta})+1} \quad \text { s.t. } \quad \bar{\theta}_{i}^{2} \leq[\boldsymbol{\Theta}]_{i i} \leq 1, \quad i=1, \ldots, R \\
\operatorname{rank}\{\boldsymbol{\Theta}\}=1, \quad \boldsymbol{\Theta} \succeq 0
\end{gathered}
$$

where $\Theta \succeq 0$ means that $\Theta$ is positive semi-definite. Introducing the auxiliary variable $t$, (28) can be written as

$$
\begin{array}{cl}
\max _{\boldsymbol{\Theta}, t} t \quad \text { s.t. } & \operatorname{tr}(\boldsymbol{\Theta}(\mathbf{Q}-t \mathbf{W})) \geq t \\
& \bar{\theta}_{i}^{2} \leq[\boldsymbol{\Theta}]_{i i} \leq 1, \quad i=1, \ldots, R \\
& \operatorname{rank}\{\boldsymbol{\Theta}\}=1, \quad \boldsymbol{\Theta} \succeq 0 .
\end{array}
$$


As the rank constraint in 29) is non-convex, this optimization problem can not be solved efficiently. Using the SDR approach (i.e., ignoring the constraint $\operatorname{rank}\{\boldsymbol{\Theta}\}=1$ in (29)), a quasi-convex optimization problem can be obtained from (29) that can be directly solved using the bisection technique [24], [38]. Based on the latter technique, the optimal value $t_{\text {opt }}$ is found in the interval $\left[t_{\text {low }}, t_{\text {up }}\right]$, where $t_{\text {low }}$ is a feasible value and therefore, $t_{\mathrm{opt}} \geq t_{\mathrm{low}}$, and $t_{\mathrm{up}}$ is not a feasible value and therefore, $t_{\mathrm{opt}} \leq t_{\mathrm{up}}$. The algorithm solves the feasibility problem

$$
\begin{array}{ll}
\text { find } \Theta \quad \text { s.t. } \quad & \operatorname{tr}(\boldsymbol{\Theta}(\mathbf{Q}-t \mathbf{W})) \geq t, \quad \boldsymbol{\Theta} \succeq 0 \\
& \bar{\theta}_{i}^{2} \leq[\boldsymbol{\Theta}]_{i i} \leq 1, \quad i=1, \ldots, R
\end{array}
$$

at the midpoint of the interval, $t=\left(t_{\text {low }}+t_{\mathrm{up}}\right) / 2$. If it is feasible, $t_{\text {low }}$ is updated as $t_{\text {low }}=t$. If it is not feasible, $t_{\mathrm{up}}$ is updated as $t_{\mathrm{up}}=t$. Then, the algorithm continues to solve the feasibility problem with the new interval until $t_{\text {up }}-t_{\text {low }}<\epsilon$, where $\epsilon$ is a parameter denoting the acceptable tolerance of the solution. The optimal matrix $\boldsymbol{\Theta}_{\text {opt }}$ is selected as $\Theta$ for the last feasible $t$, (i.e., $t=t_{\text {low }}$ in the last step). If the matrix $\Theta_{\mathrm{opt}}$ is rank-one, then its principal eigenvector is the optimal solution to (26). If $\Theta_{\mathrm{opt}}$ is not rank-one, then a proper approximate solution for $\boldsymbol{\theta}$ can be obtained using randomization techniques [39].

\section{Extended Distributed Alamouti Code}

The scheme developed in the previous section applies to the case of $T=1$. In what follows, we extend it to the case of $T=2$ by developing an approach based on the distributed Alamouti code to reduce the total feedback rate. Using computer simulations, the latter scheme will be shown to provide robustness against feedback errors. Such improvements in the feedback rate and robustness are, however, achieved at the price of an increased decoding delay and a moderate performance loss as compared to the case of $T=1$.

Let us consider the case of an even number of relayd2, i.e., let $R=2 K$ where $K$ is some positive integer. The distributed Alamouti code is used by relay pairs. Let each $k$ th relay pair receive a low-rate feedback to select the binary coefficient $b_{k} \in\{-1,1\}$ and the real-valued power loading coefficient $\theta_{k} \in[0,1]$. Since the same $b_{k}$ and $\theta_{k}$ should be used by the two relays of the $k$ th relay pair, the receiver can broadcast them to both these relays, thereby reducing the feedback rate almost by half.

\footnotetext{
${ }^{2}$ The case of an odd number of relays can be addressed in the same way and, therefore, is omitted below.
} 
The relays use the basic distributed Alamouti code matrices [14] to form the signal transmitted by each relay pair as: $\tilde{\mathbf{A}}_{2 k-1}=\mathbf{I}_{2}\left(\right.$ with $\mathbf{B}_{2 k-1}=\mathbf{O}$ ) and $\tilde{\mathbf{A}}_{2 k}=\left[\begin{array}{rr}0 & -1 \\ 1 & 0\end{array}\right]$ with $\mathbf{A}_{2 k}=\mathbf{O}$, where $\mathbf{I}_{2}$ is the $2 \times 2$ identity matrix. Using (6)-(8), we obtain the following distributed space-time code matrix for the proposed scheme:

$$
\mathbf{S}=\left[\mathbf{S}_{a}, \cdots, \mathbf{S}_{a}\right]
$$

where $\mathbf{S}_{a}=\left[\begin{array}{rr}s_{1} & -s_{2}^{*} \\ s_{2} & s_{1}^{*}\end{array}\right]$ is the conventional Alamouti code matrix.

The channel and relay power vectors are given by

$$
\begin{aligned}
& \mathbf{h}=\left[f_{1} g_{1}, f_{2}^{*} g_{2}, \ldots, f_{2 K-1} g_{2 K-1}, f_{2 K}^{*} g_{2 K}\right]^{T} \\
& \mathbf{p}=\left[p_{1}, p_{2}, \ldots, p_{2 K-1}, p_{2 K}\right]^{T}=\left[\sqrt{\frac{P_{0} P_{1} T}{m_{f_{1}} P_{0}+1}} b_{1} \theta_{1}, \ldots, \sqrt{\frac{P_{0} P_{2 K} T}{m_{f_{2 K} P_{0}+1}}} b_{K} \theta_{K}\right]^{T}
\end{aligned}
$$

respectively.

Note that in contrast to $[8]$, any $(2 k-1)$ th and $(2 k)$ th relays use the same $b_{k} \theta_{k}$.

Conjugating the second entry $x_{2}$ of the vector $\mathbf{x}=\left[x_{1}, x_{2}\right]^{T}$ in (6) and using (31)-(33), we obtain the following equivalent model

$$
\breve{\mathbf{x}}=\mathbf{H} \breve{\mathbf{s}}+\breve{\mathbf{w}}
$$

where $\breve{\mathbf{x}} \triangleq\left[x_{1}, x_{2}^{*}\right]^{T}, \breve{\mathbf{s}} \triangleq\left[s_{1}, s_{2}^{*}\right]^{T}, \breve{\mathbf{w}} \triangleq\left[w_{1}, w_{2}^{*}\right]^{T}$,

$$
\mathbf{H}=\sum_{k=1}^{K} \mathbf{H}_{k} \quad \text { and } \quad \mathbf{H}_{k}=\left[\begin{array}{cc}
p_{2 k-1} h_{2 k-1} & -p_{2 k} h_{2 k} \\
p_{2 k} h_{2 k}^{*} & p_{2 k-1} h_{2 k-1}^{*}
\end{array}\right] .
$$

Note that

$$
\mathbf{H}^{H} \mathbf{H}=\left[\begin{array}{cc}
\gamma_{a}+\beta_{a} & 0 \\
0 & \gamma_{a}+\beta_{a}
\end{array}\right]
$$

where

$$
\begin{aligned}
& \gamma_{a} \triangleq\|(\mathbf{p} \odot \mathbf{h})\|^{2} \\
& \beta_{a} \triangleq \sum_{i, j=1, i \neq j}^{K} \theta_{i} b_{i} \theta_{j} b_{j} \operatorname{Re}\left\{\rho_{2 i-1,2 j-1} h_{2 i-1} h_{2 j-1}^{*}+\rho_{2 i, 2 j} h_{2 i} h_{2 j}^{*}\right\} .
\end{aligned}
$$

Throughout (31)-(36), the subindex $(\cdot)_{a}$ stands for the extended Alamouti scheme.

Since the matrices $\tilde{\mathbf{A}}_{i}$ satisfy the property $\tilde{\mathbf{A}}_{i} \tilde{\mathbf{A}}_{i}^{H}=\mathbf{I}_{T}$, the noise covariance matrix $\mathbf{R}_{\breve{\mathbf{w}}} \triangleq \mathrm{E}\left\{\breve{\mathbf{w}} \breve{\mathbf{w}}^{H}\right\}$ is a scaled identity matrix. Therefore, the ML decoding

$$
\arg \min _{\breve{\mathbf{s}}}\|\breve{\mathbf{x}}-\mathbf{H} \breve{\mathbf{s}}\|
$$


reduces to simple symbol-by-symbol decoding.

As $\left|h_{k}\right|^{2}=\left|\tilde{f}_{k} g_{k}\right|^{2}$, it is clear from (35), (36) and Proposition 1 that the maximum diversity can be achieved if $\beta_{a} \geq 0$. Similar to [32], it can be proved that if $b_{k} \in\{-1,1\}$, it can be guaranteed that $\beta_{a} \geq 0$.

As in Section III, the coefficients $b_{k}$ can be selected using the exhaustive full search, a suboptimal SDR approach similar to Algorithm 1, or an iterative procedure similar to Algorithm 2. To develop the SDR approach for the extended distributed Alamouti code case, we define the $K \times 1$ vector $\mathbf{b}_{a} \triangleq\left[b_{1}, \ldots, b_{K}\right]^{T}$ and the $2 \times K$ matrix

$$
\mathbf{F} \triangleq\left[\begin{array}{cccc}
p_{1} h_{1} & p_{3} h_{3} & \cdots & p_{2 K-1} h_{2 K-1} \\
p_{2} h_{2} & p_{4} h_{4} & \cdots & p_{2 K} h_{2 K}
\end{array}\right]
$$

Using (37), we obtain that

$$
\gamma_{a}+\beta_{a}=\mathbf{b}_{a}^{T} \mathbf{F}^{H} \mathbf{F} \mathbf{b}_{a}
$$

Defining $\overline{\mathbf{Q}}_{a} \triangleq \mathbf{F}^{H} \mathbf{F}$, we can write the problem of optimal selection of the coefficients $b_{k}(k=1, \ldots, K)$ as

$$
\max _{\mathbf{b}_{a} \in\{-1,1\}^{K}} \mathbf{b}_{a}^{T} \overline{\mathbf{Q}}_{a} \mathbf{b}_{a}
$$

Using the notation $\mathbf{B}_{a} \triangleq \mathbf{b}_{a} \mathbf{b}_{a}^{T}$, this problem can be rewritten as

$$
\begin{aligned}
\max _{\mathbf{B}_{a}} \operatorname{tr}\left(\mathbf{B}_{a} \overline{\mathbf{Q}}_{a}\right) \quad \text { s.t. } & \operatorname{rank}\left\{\mathbf{B}_{a}\right\}=1, \quad \mathbf{B}_{a} \succeq 0 \\
& {\left[\mathbf{B}_{a}\right]_{k k}=1, \quad k=1, \ldots, K }
\end{aligned}
$$

and using the SDR approach, it can be approximately converted to a convex form

$$
\max _{\mathbf{B}_{a}} \operatorname{tr}\left(\mathbf{B}_{a} \overline{\mathbf{Q}}_{a}\right) \text { s.t. } \mathbf{B}_{a} \succeq 0, \quad\left[\mathbf{B}_{a}\right]_{k k}=1, k=1, \ldots, K
$$

by omitting the rank-one constraint $\operatorname{rank}\left\{\mathbf{B}_{a}\right\}=1$ in [39].

The SDR-based algorithm for the proposed distributed Alamouti approach be summarized as follows. Algorithm 3

1. At the receiver, find the solution to (40) using the approach of [37].

2. Send the so-obtained $b_{k}$ from the receiver to the $k$ th relay pair for each $k=1, \ldots, K$ using one-bit per relay pair feedback.

In turn, the greedy algorithm of Section $\amalg$ can be modified as follows.

\section{Algorithm 4}

1. Set $b_{1}=1$ and $\tau_{1}=\left[h_{1} h_{2}\right]^{T}$. 
2. For $k=2, \ldots, K$, compute

$$
b_{k}=\operatorname{sign}\left(\operatorname{Re}\left\{\left[h_{2 k-1}^{*} h_{2 k}^{*}\right] \tau_{k-1}\right\}\right), \quad \tau_{k}=\tau_{k-1}+b_{k}\left[h_{2 k-1} h_{2 k}\right]^{T} .
$$

3. Send the so-obtained $b_{k}$ from the receiver to the $k$ th relay pair for each $k=1, \ldots, K$ using one-bit per relay pair feedback.

To derive the power loading coefficients $\theta_{k}$, an approach similar to that presented in Section III-B can be applied. We first develop an approximation to the expected value of the signal power and then maximize a lower bound on the SNR. Using (34), the average signal power can be written as

$$
\mathrm{E}\left\{P_{s}\right\}=\mathrm{E}\left\{\gamma_{a}\right\}+\mathrm{E}\left\{\beta_{a}\right\}
$$

Using (35), (36) and the same arguments as in Section $\amalg$ II-B $\mathrm{E}\left\{P_{s}\right\}$ can be approximated as

$$
\mathrm{E}\left\{P_{s}\right\} \approx \sum_{i, j=1}^{K} \theta_{i} \theta_{j}\left|\operatorname{Re}\left\{\mathrm{E}\left\{\rho_{2 i-1,2 j-1} h_{2 i-1} h_{2 j-1}^{*}+\rho_{2 i, 2 j} h_{2 i} h_{2 j}^{*}\right\}\right\}\right|
$$

where it is assumed that the optimal values of $b_{i}(i=1, \ldots, K)$ are selected.

The expected value of the noise is given by

$$
\mathrm{E}\left\{P_{w}\right\}=1+\sum_{k=1}^{K} \theta_{k}^{2}\left(\frac{P_{2 k-1} m_{g_{2 k-1}}}{P_{0} m_{f_{2 k-1}}+1}+\frac{P_{2 k} m_{g_{2 k}}}{P_{0} m_{f_{2 k}}+1}\right) .
$$

Using (42) and (43), the SNR maximization problem can be approximated as

$$
\max _{\boldsymbol{\theta}_{a}} \frac{\boldsymbol{\theta}_{a}^{T} \mathbf{Q}_{a} \boldsymbol{\theta}_{a}}{\boldsymbol{\theta}_{a}^{T} \mathbf{W}_{a} \boldsymbol{\theta}_{a}+1} \quad \text { s.t. } \quad \bar{\theta}_{k} \leq \theta_{k}^{2} \leq 1, i=1, \ldots, K
$$

where $\boldsymbol{\theta}_{a} \triangleq\left[\theta_{1} \ldots \theta_{K}\right]^{T}$,

$$
\mathbf{W}_{a} \triangleq \operatorname{diag}\left(\sum_{l=1}^{2} \frac{m_{g_{l}} P_{l}}{m_{f_{l}} P_{0}+1}, \ldots, \sum_{l=2 K-1}^{2 K} \frac{m_{g_{l}} P_{l}}{m_{f_{l}} P_{0}+1}\right)
$$

$\mathbf{Q}_{a}$ is a $K \times K$ matrix with the entries

$$
\left[\mathbf{Q}_{a}\right]_{i, j} \triangleq\left|\operatorname{Re}\left\{\rho_{2 i-1,2 j-1} \mathrm{E}\left\{h_{2 i-1} h_{2 j-1}^{*}\right\}+\rho_{2 i, 2 j} \mathrm{E}\left\{h_{2 i} h_{2 j}^{*}\right\}\right\}\right|
$$

and $\bar{\theta}_{k}$ constrains the coefficients $\theta_{k}$ to prevent diversity losses in a way similar to that described in Section III] Now, the expected value in 45) can be estimated using the statistical independence of the channels as in (22). In particular, for the $(2 i, 2 j)$ th factor in (45), we have

$$
\mathrm{E}\left\{h_{2 i} h_{2 j}^{*}\right\}=\left(\mu_{f_{2 i}} \mu_{f_{2 j}}^{*}+\delta_{(2 i)(2 j)} \sigma_{f_{2 i}}^{2}\right)\left(\mu_{g_{2 i}} \mu_{g_{2 j}}^{*}+\delta_{(2 i)(2 j)} \sigma_{g_{2 i}}^{2}\right) .
$$

Following the same steps as in Section III the optimization problem in (44) can be turned into a convex feasibility problem that extends (30) to the distributed Alamouti coding case. 


\section{DifFERENTIAL TRANSMISSION}

The concept of differential transmission is used in this section to extend the proposed approach to the case where no CSI is available at the receiver. Let us assume that $T=1$ and let the transmitter encode differentially the information symbols $s_{l}$ selected from some constant-modulo constellation $\mathcal{S}$ as

$$
u_{l}=u_{l-1} s_{l}, \quad u_{0}=1
$$

where $u_{l}$ and $u_{0}$ are the current and initial transmitted symbols, respectively. Similar to the coherent scheme in (9), we have

$$
x_{l}=\mathbf{1}_{R}^{T}(\mathbf{p} \odot \mathbf{h}) u_{l}+w_{l} .
$$

Using (46) and the previous received signal $x_{l-1}$, the ML symbol estimate can be obtained from maximizing [1]

$$
\operatorname{Re}\left\{x_{l-1} x_{l}^{*} s_{l}\right\}
$$

over $s_{l} \in \mathcal{S}$. We assume that no power loading is used, i.e., set $\theta_{i}=1$ for $i=1, \ldots, R$. Since the receiver has no CSI to select the feedback bits for $b_{i}(i=1, \ldots, R)$, the following simple sequential feedback bit assignment scheme can be used. Before the beginning of the frame in which the information symbols should be transmitted, there is an extra transmission stage to select the coefficients $b_{i}$. First, $u_{0}$ is transmitted from the source to the relays and then it is retransmitted by the relays to the destination with $b_{i}=1(i=1, \ldots, R)$. Then, the second relay only alters its coefficient $b_{2}$ to -1 and the relays retransmit again. The received powers corresponding to the latter two relay-to-destination transmissions are compared at the receiver and the receiver sends one bit of feedback. This bit is used by the second relay to select $b_{2}$ that corresponds to the maximum received power. The process continues with the remaining relays in the same way. This makes it possible to select all the coefficients $b_{i}(i=2, \ldots, R)$ in a sequential (greedy) way. After the process of selecting the coefficients $b_{i}$ is completed, the source starts the transmission of its information symbols. The overall transmission strategy can be summarized as follows:

\section{Algorithm 5}

1. Set $b_{i}=1, i=1, \ldots, R$. Transmit $u_{0}$ from the source to relays and then retransmit it from the relays to the destination to obtain $x_{1}=\mathbf{1}_{R}^{T}(\mathbf{p} \odot \mathbf{h}) u_{0}+w_{1}$ at the receiver.

2. For $j=2, \ldots, R$ :

- At the $j$ th relay, set $b_{j}=-1$ and, using (3), update the signal $d_{j}$ to be transmitted from this particular relay. 
- Transmit signals from all relays to obtain $x_{j}=\mathbf{1}_{R}^{T}(\mathbf{p} \odot \mathbf{h}) u_{0}+w_{j}$ at the receiver.

- If $\left|x_{j}\right|^{2}>\left|x_{j-1}\right|^{2}$, then feed " 1 " from the receiver back to the relay; otherwise feed " 0 " back to the relay. In the latter case, set $x_{j}=x_{j-1}$.

- If the received feedback at the $j$ th relay is 1 , then select $b_{j}=-1$. Otherwise, select $b_{j}=1$.

Similarly, a differential modification of the extended distributed Alamouti code of Section IV can be developed in the case when $T=2$. At the transmitter, a unitary matrix $\mathbf{S}_{l}$ should be formed from the constant-modulo information symbols $s_{2 l-1}, s_{2 l}$ as

$$
\mathbf{S}_{l}=\frac{1}{\sqrt{2}}\left[\begin{array}{cc}
s_{2 l-1} & -s_{2 l}^{*} \\
s_{2 l} & s_{2 l-1}^{*}
\end{array}\right] .
$$

Let the differential encoding

$$
\mathbf{u}_{l}=\mathbf{S}_{l} \mathbf{u}_{l-1}
$$

be used at the transmitter. It amounts to sending the vector $\mathbf{u}_{l}=\left[u_{2 l-1}, u_{2 l}\right]^{T}$ instead of $\mathbf{s}_{l}=\left[s_{2 l-1}, s_{2 l-1}\right]^{T}$ to the relays where $l$ denotes the transmitted block number. The first vector $\mathbf{u}_{l}$ can be chosen as $\mathbf{u}_{0}=[1,0]^{T}$. Similar to (6) and using the matrices $\tilde{\mathbf{A}}_{2 k-1}$ and $\tilde{\mathbf{A}}_{2 k}$ defined in the previous section for the extended distributed Alamouti code, the following equivalent relation can be obtained

$$
\mathbf{x}_{l}=\mathbf{S}_{l} \mathbf{U}_{l-1}\left(\sum_{k=1}^{K} \mathbf{p}_{k} \odot \mathbf{h}_{k}\right)+\mathbf{w}_{l}
$$

where $\mathbf{U}_{0}=\mathbf{I}_{2}$,

$$
\begin{aligned}
\mathbf{U}_{l-1} & \triangleq\left[\begin{array}{cc}
u_{2 l-3} & -u_{2 l-2}^{*} \\
u_{2 l-2} & u_{2 l-3}^{*}
\end{array}\right], \quad l>1 \\
\mathbf{p}_{k} & \triangleq\left[\sqrt{\frac{P_{0} P_{2 k-1} T}{m_{f_{2 k-1}} P_{0}+1}} b_{k} \theta_{k}, \sqrt{\frac{P_{0} P_{2 k} T}{m_{f_{2 k}} P_{0}+1}} b_{k} \theta_{k}\right]^{T} \\
\mathbf{h}_{k} & \triangleq\left[f_{2 k-1} g_{2 k-1}, f_{2 k}^{*} g_{2 k}\right]^{T} .
\end{aligned}
$$

The ML decoding amounts to maximizing [1]

$$
\operatorname{Re}\left\{\operatorname{tr}\left(\mathbf{x}_{l-1} \mathbf{x}_{l}^{H} \mathbf{S}_{l}\right)\right\}
$$

over $s_{2 l-1}, s_{2 l} \in \mathcal{S}$. Note that the detection can be done symbol-by-symbol. As in the previous scheme without DSTC, we set $\theta_{i}=1$ and use a similar strategy to select the coefficients $b_{i}$ using relay pairs and blocks of length $T=2$. This strategy can be summarized as follows:

Algorithm 6 
1. Set $b_{i}=1, i=1, \ldots, K$. Transmit $\mathbf{u}_{0}$ from the source to relays and then retransmit it from the relays to the destination to obtain $\mathbf{x}_{1}=\mathbf{U}_{0}\left(\sum_{k=1}^{K} \mathbf{p}_{k} \odot \mathbf{h}_{k}\right)+\mathbf{w}_{1}$ at the receiver.

2. For $j=2, \ldots, K$ :

- At the $(2 j-1)$ th and $(2 j)$ th relays, set $b_{j}=-1$ and, using (3), update the signals $\mathbf{d}_{2 j-1}$ and $\mathbf{d}_{2 j}$ to be transmitted from this particular relay pair.

- Transmit signals from all relays to obtain $\mathbf{x}_{j}=\mathbf{U}_{0}\left(\sum_{k=1}^{K} \mathbf{p}_{k} \odot \mathbf{h}_{k}\right)+\mathbf{w}_{j}$ at the receiver.

- If $\left\|\mathbf{x}_{j}\right\|^{2} \geq\left\|\mathbf{x}_{j-1}\right\|^{2}$, then feed " 1 " from the receiver back to the relay; otherwise feed " 0 " back to the relay. In the latter case, set $\mathbf{x}_{j}=\mathbf{x}_{j-1}$.

- If the received feedback at the $(2 j-1)$ th and $(2 j)$ th relays is 1 then select $b_{j}=-1$. Otherwise, select $b_{j}=1$.

Similar to Algorithms 2 and 4, Algorithms 5 and 6 are suboptimal. However, the latter two algorithms do not require any CSI at the receiver and, moreover, our simulations demonstrate that they achieve maximum diversity. It is also worth noting that this diversity benefit is achieved at linear decoding complexity.

\section{Simulations}

Throughout our simulation examples, the QPSK modulation is used and the channels are assumed to be statistically independent from each other. In all but the fourth example, we consider all the channels to be complex circular Gaussian random variables with zero-mean and unit variance and assume that $\theta_{i}=1, i=1, \ldots, R$ (which are the optimal power loading coefficients in this case). For the sake of fairness of our comparisons, only techniques that do not need the instantaneous CSI at the relays are tested. Unless specified otherwise, the feedback is considered to be error-free.

In the first example, we compare the bit error rate (BER) performances of the algorithms that select the coefficients $b_{i}$ using the cooperative transmission scheme of Section $\amalg$ II-A with $R=20$ relays and the same maximum power $P_{0}=\ldots=P_{R}=P /(R+1)$. In this example, the full search-based (optimal) algorithm is compared with Algorithms 1 and 2. Fig. 2 displays the BERs of these algorithms $P / \sigma^{2}$. It can be seen from this figure that the SDR-based approach (Algorithm 1) performs about $1 \mathrm{~dB}$ better than the iterative procedure of Algorithm 2. The performances of the optimal full search algorithm and Algorithm 1 are nearly identical.

In our second example, the performances of the cooperative transmission schemes of Sections III-A (Algorithm 1) and IV (Algorithm 4) are compared with that of the best relay selection (BRS) scheme, the distributed beamforming approach of [27] with quantized feedback, and the distributed version of the 
QOSTBC [14]. In the BRS scheme, the destination selects the relay that enjoys the largest receive SNR. The relays only have the knowledge of their average receive power $\mathrm{E}\left\{\left|r_{i}\right|^{2}\right\}=m_{f_{i}} P_{0}+1$ and they use this knowledge to normalize the transmitted signal so that the average transmitted power of the $i$ th relay is $P_{i}$. It can be readily shown that this corresponds to the following relay selection rule at the destination:

$$
\arg \max _{i=1, \ldots, R} \frac{\left|f_{i} g_{i}\right|^{2} P_{i}}{1+m_{f_{i}} P_{0}+\left|g_{i}\right|^{2} P_{i}} \text {. }
$$

Throughout this example, $R=4$ and the source and relay powers are chosen from the optimal power distribution for DSTC [14] as $P_{0}=P / 2$ and $P_{i}=P /(2 R)(i=1, \ldots, R)$. For the sake of fairness, the distributed beamforming algorithm of [27] was implemented without the knowledge of the instantaneous channel $f_{i}$ at each $i$ th relay using the generalized Lloyd and genetic algorithms. The beamformer codebooks required in the technique of [27] have been designed for the cases of one and three feedback bits. Fig. 3 displays the BERs of the techniques tested versus $P / \sigma^{2}$.

Note that the distributed QOSTBC technique does not require any feedback, whereas the BRS technique requires two bits of feedback, and the Algorithms 1 and 4 require three and one bits of feedback, respectively. However, the distributed QOSTBC approach requires a more complicated decoder and imposes the decoding delay of $T=4$.

It can be clearly seen from this figure that both Algorithms 1 and 4 substantially outperform BRS, distributed QOSTBC, and the distributed beamforming approach of [27] with one-bit feedback. Also, Algorithm 1 outperforms Algorithm 4 with the performance gain of more than $2 \mathrm{~dB}$ at the cost of a higher feedback rate. The performances of Algorithm 1 and the approach of [27] with three bits of feedback are nearly identical. However, it should be noted that the codebook design in the technique of [27] represents a rather difficult optimization problem, and that this codebook has to be completely redesigned and resent to the relay nodes whenever the channel statistics or the transmitted powers change. This makes the implementation of the beamformer of [27] substantially more difficult than that of our algorithms.

Fig. 4 compares the performance of our Algorithms 1 and 4 with that of the BRS technique and the beamformer of [27] in the erroneous feedback case. All the other parameters are the same as used in the previous figure. From Fig. 4 we observe that our algorithms are less sensitive to feedback errors than BRS and the approach of [27].

In our third example, $R=4$ is chosen. In this example, the performance of the differential techniques developed in Section $\nabla$ is compared to that of the $\mathrm{Sp}(2) \mathrm{DSTC}$ of [40], the coherent distributed QOSTBC 
of [14] and the BRS technique with differential transmission in which the relay with the largest received power is selected. Note that both the $\mathrm{Sp}(2)$ DSTC and the coherent distributed QOSTBC do not require any feedback, whereas the BRS approach requires a total of two feedback bits. It should be also stressed that unlike our differential schemes and the other schemes considered in this example, the coherent distributed QOSTBC requires full CSI at the receiver. The symbol rates of the $\mathrm{Sp}(2) \mathrm{DSTC}$, the coherent distributed QOSTBC and the BRS technique are the same as that of our differential techniques and are equal to $1 / 2$ symbols per channel use. Important advantages of our technique w.r.t. the $\operatorname{Sp}(2)$ DSTC are lower decoding complexity, shorter required channel coherence time, and lower decoding delay.

For the $\mathrm{Sp}(2)$ code, we use the 3-PSK constellation for the first two symbols and the 5-PSK constellation for the other two symbols. With that, a total rate of 0.9767 bits per channel use (bpcu) is achieved. The other schemes use the QPSK symbol constellations to achieve the total rate of $1 \mathrm{bpcu}$.

Fig. 5 compares the block error rate (BLER) performance of the techniques evaluated versus $P / \sigma^{2}$. The values of BLER are computed using blocks of four symbols. As can be observed from Fig. 5, both Algorithms 5 and 6 outperform the $\mathrm{Sp}(2)$ code and the differential BRS approach, and their performance is close to the distributed QOSTBC (which requires the full CSI knowledge). In particular, it can be seen from this figure that the proposed techniques have approximately the same (maximum) diversity order as the $\mathrm{Sp}(2)$ code and the distributed QOSTBC with coherent decoder.

These improvements come at the price of three bits and one bit of feedback for Algorithms 5 and 6, respectively. Also, note that Algorithm 5 uses a total of $2 R$ auxiliary time-slots before starting the transmission of information bits, while Algorithm 6 uses $3 K+1$ time-slots (one time-slot for each feedback bit). On the other hand, the $\mathrm{Sp}(2)$ code uses $2 R$ auxiliary time-slots.

In our fourth example, the performance of Algorithm 1 combined with long-term power loading (which is developed in Section $\amalg$ II-B is compared with Algorithm 1 of Section $\amalg I-A$ and with the analytical results obtained from (48) by means of brute force optimization. For long-term power loading, the approach of (30) with bisection search is used. In this example, $R=4$ and $\bar{\theta}_{1}=\ldots=\bar{\theta}_{R} \triangleq \bar{\theta}$, where the nearly optimal value of $\bar{\theta}=0.1$ has been chosen. The relay locations have been uniformly drawn from a circle of normalized radius 0.5 , while the distance between the source and destination is equal to 2; see Fig. 6 that explicitly clarifies the geometry. The values of $m_{f_{i}}$ and $m_{g_{i}}$ depend on the distance from the transmitter to the $i$ th relay, where $m_{f_{i}}=m_{g_{i}}=1$ in the center of the circle. We assume that the path-loss exponent is equal to 3 . The performance is averaged over random channel realizations whereas the relay locations are kept fixed over all simulation runs. Both the line-of-sight (LOS) and nonLOS (NLOS) scenarios are considered and equal maximum powers of the transmitter and relay nodes 
$\left(P_{0}=P_{1} \ldots=P_{R}=P /(R+1)\right)$ are taken. In the LOS channel case, it is assumed that $\phi_{f_{i}}=\phi_{g_{i}}=1$ where $\phi_{f_{i}} \triangleq\left|\mu_{f_{i}}\right|^{2} / \sigma_{f_{i}}^{2}$ and $\phi_{g_{i}} \triangleq\left|\mu_{g_{i}}\right|^{2} / \sigma_{g_{i}}^{2}$.

In Fig. 7 the BERs of the algorithms tested are shown versus $P / \sigma^{2}$. As can be clearly seen from the figure, the proposed approach with long-term power loading achieves nearly the same performance as predicted by (48) and substantially outperforms Algorithm 1 without power loading.

In our fifth example, we compare the performances of Algorithm 1 of Section $\amalg-A$ and Algorithm 4 of Section IV in the cases of perfect and imperfect feedback. In this example, $R=4, P_{0}=P_{1} \ldots=$ $P_{R}=P /(R+1)$ and the feedback error probabilities $P_{e}=10^{-2}$ and $P_{e}=10^{-3}$ are assumed.

Fig. 8 displays the BERs of the methods evaluated versus $P / \sigma^{2}$. As can be observed from this figure, the performance of Algorithm 1 becomes sensitive to feedback errors when the BER values are smaller than the feedback error probability itself. Therefore, as the same link quality can be normally expected in both directions, the performance of Algorithm 1 should not be significantly affected by feedback errors.

It can also be seen from Fig. 8 that, in contrast to Algorithm 1, the performance of Algorithm 4 is not sensitive to feedback errors. The latter fact can be explained by the spatial diversity of the Alamouti code.

\section{CONCLUSIONS}

A new approach to the use of a low-rate feedback in wireless relay networks has been proposed. It has been shown that our scheme achieves the maximum possible diversity offered by the relay network. To further improve the performance of the proposed scheme in practical scenarios, the knowledge of second-order channel statistics has been used to obtain long-term power loading coefficients by means of maximizing the receiver signal-to-noise ratio with proper power constraints. This maximization problem has been shown to be approximately equivalent to a convex feasibility problem whose solution has been demonstrated to be close to the optimal one in terms of the error probability. To improve the robustness of our scheme against feedback errors and further decrease the feedback rate, an extended version of the distributed Alamouti code has been developed. Finally, extensions of the proposed approach to the differential transmission case have been discussed.

Simulations have verified an improved performance-to-feedback tradeoff of the proposed techniques as compared to other popular techniques such as distributed QOSTBC of [14], best relay selection method, distributed beamforming technique of [27] with quantized feedback, and the $S p(2)$ distributed code of [40]. 


\section{APPENDIX}

\section{PROOF OF PROPOSITION 1}

The symbol error probability (SER) for (9) is given by [41]

$$
\mathrm{SER}=c_{1} Q\left(\sqrt{c_{2} \frac{P_{s}}{P_{w}}}\right)
$$

where $c_{1}$ and $c_{2}$ are two constants that depend on the constellation used, and $Q(x)=\frac{1}{2 \pi} \int_{x}^{\infty} e^{-t^{2} / 2} d t$.

Using the Chernoff bound, we have

$$
\overline{\mathrm{SER}} \leq \frac{c_{1}}{2} \mathrm{E}_{f_{i}, g_{i}}\left\{e^{-c_{2} \frac{P_{s}}{2 P_{w}}}\right\} .
$$

Note that if we establish an upper bound for $\beta=0$, then it will be also valid for any $\beta>0$. This follows from the fact that $Q(x)$ is a monotonically decreasing function. Using this fact, let us obtain an upper bound on $\overline{\mathrm{SER}}$ by using the particular value $\beta=0$. Then, from (48) we obtain

$$
\overline{\mathrm{SER}} \leq \frac{c_{1}}{2} \mathrm{E}_{f_{i}, g_{i}}\left\{e^{-c_{2} \frac{\gamma}{2 P_{w}}}\right\} .
$$

First, let us calculate the expected value over the channel coefficients $f_{i}$. As these coefficients are statistically independent, each term in the sum for $\gamma$ can be calculated independently. Using the complex Gaussian pdf for $f_{i}$

$$
p_{f_{i}}\left(f_{i}\right)=\frac{1}{\pi \sigma_{f_{i}}^{2}} e^{-\left|f_{i}-\mu_{f_{i}}\right|^{2} / \sigma_{f_{i}}^{2}}
$$

and defining

$$
a_{i} \triangleq \frac{c_{2}\left|g_{i}\right|^{2} \rho_{i, i}}{2 P_{w}}
$$

we obtain from (49) that

$$
\overline{\mathrm{SER}} \leq \frac{c_{1}}{2} \mathrm{E}_{g_{i}}\left\{\prod_{i=1}^{R} \Upsilon_{i}\right\}
$$

where

$$
\Upsilon_{i} \triangleq \frac{1}{\pi \sigma_{f_{i}}^{2}} \int_{-\infty}^{\infty} e^{-a_{i}\left|f_{i}\right|^{2}-\left|f_{i}-\mu_{f_{i}}\right|^{2} / \sigma_{f_{i}}^{2}} d f_{i}
$$

After straightforward manipulations, (53) can be rewritten as

$$
\Upsilon_{i}=\frac{1}{a_{i} \sigma_{f_{i}}^{2}+1} e^{-\frac{\left|\mu_{f_{i}}\right|^{2}}{\sigma_{f_{i}}}\left(\frac{a_{i} \sigma_{f_{i}}^{2}}{a_{i} \sigma_{f_{i}}^{2}+1}\right)} \int_{-\infty}^{\infty} \frac{a_{i} \sigma_{f_{i}}^{2}+1}{\pi \sigma_{f_{i}}^{2}} e^{-\frac{\left(a_{i} \sigma_{f_{i}}^{2}+1\right)}{\sigma_{f_{i}}^{2}}\left|f_{i}-\frac{\mu_{f_{i}}}{\left(a_{i} \sigma_{f_{i}}^{2}+1\right)}\right|^{2}} d f_{i} .
$$

The function inside the integral in (54) is equal to the complex Gaussian pdf $\mathcal{C N}\left(\frac{\mu_{f_{i}}}{a_{i} \sigma_{f_{i}}^{2}+1}, \frac{\sigma_{f_{i}}^{2}}{a_{i} \sigma_{f_{i}}^{2}+1}\right)$. Therefore, the integral in (54) is equal to one and we obtain that

$$
\Upsilon_{i}=\frac{1}{a_{i} \sigma_{f_{i}}^{2}+1} e^{-\phi_{f_{i}}\left(\frac{a_{i} \sigma_{f_{i}}^{2}}{a_{i} \sigma_{f_{i}}^{2}+1}\right)}
$$


where $\phi_{f_{i}}=\left|\mu_{f_{i}}\right|^{2} / \sigma_{f_{i}}^{2}$. An upper bound approximation for the expected value in (52) can be derived as follows. Since $a_{i} \geq 0$, we have that $0 \leq \frac{a_{i} \sigma_{f_{i}}^{2}}{a_{i} \sigma_{f_{i}}^{2}+1}<1$. Therefore, $\Upsilon_{i}$ can be upper-bounded as $\Upsilon_{i} \leq 1 /\left(a_{i} \sigma_{f_{i}}^{2}+1\right)$ and

$$
\overline{\mathrm{SER}} \leq \frac{c_{1}}{2} \mathrm{E}_{g_{i}}\left\{\prod_{i=1}^{R} \frac{1}{a_{i} \sigma_{f_{i}}^{2}+1}\right\} .
$$

Let us characterize the power of each transmitting node $P_{i}(i=0, \ldots, R)$ as a fraction $P_{i}=\lambda_{i} P$ of the total power $P=\sum_{i=0}^{R} P_{i}$, where $\sum_{i=0}^{R} \lambda_{i}=1$. If $R$ is large, then, according to the law of large numbers,

$$
\sum_{i=1}^{R} \frac{\lambda_{i}\left|g_{i}\right|^{2}}{m_{f_{i}} \lambda_{0}+P^{-1}} \leq R \alpha
$$

where the inequality is satisfied in the almost sure sense,

$$
\alpha \triangleq \max _{i=1, \ldots, R}\left(\frac{\lambda_{i} m_{g_{i}}}{m_{f_{i}} \lambda_{0}+P^{-1}}\right)
$$

and $m_{g_{i}} \triangleq \mathrm{E}\left\{\left|g_{i}\right|^{2}\right\}=\left|\mu_{g_{i}}\right|^{2}+\sigma_{g_{i}}^{2}$. Therefore, from (10) and (51), we have that

$$
\frac{1}{a_{i} \sigma_{f_{i}}^{2}+1}=\frac{1}{\frac{c_{2} \sigma_{f_{i}}^{2}\left|g_{i}\right|^{2} \rho_{i, i}}{2 P_{w}}+1} \leq \frac{1}{\frac{c_{2} \sigma_{f_{i}}^{2}\left|g_{i}\right|^{2} \rho_{i, i}}{2(1+R \alpha)}+1} .
$$

Using (12) and (56), from (55) we obtain that

$$
\overline{\mathrm{SER}} \leq \frac{c_{1}}{2} \mathrm{E}_{g_{i}}\left\{\prod_{i=1}^{R} \frac{1}{\bar{a}_{i} P\left|g_{i}\right|^{2}+1}\right\}
$$

where

$$
\bar{a}_{i} \triangleq \frac{c_{2} \sigma_{f_{i}}^{2} \lambda_{0} \lambda_{i}}{2\left(m_{f_{i}} \lambda_{0}+1 / P\right)(1+R \alpha)} .
$$

From (57) and the fact that all the channel coefficients are statistically independent, it can be readily seen that for each $i$ the expectation over $g_{i}$ in the right-hand side can be calculated independently from the other values $g_{l}, l \neq i$. The random variable $z_{i}=\left|g_{i}\right|^{2}$ has the non-central chi-square pdf with two degrees of freedom:

$$
p_{\left|g_{i}\right|^{2}}\left(z_{i}\right)=\frac{1}{\sigma_{g_{i}}^{2}} e^{-\frac{z_{i}+\left|\mu_{g_{i}}\right|^{2}}{\sigma_{g_{i}}^{2}}} I_{0}\left(\frac{2\left|\mu_{g_{i}}\right| \sqrt{z_{i}}}{\sigma_{g_{i}}^{2}}\right)
$$

where $I_{0}(\cdot)$ denotes the modified zero-order Bessel function of the first kind. Using (58) to compute the expectation in (57), we have

$$
\overline{\mathrm{SER}} \leq \frac{c_{1}}{2} \prod_{i=1}^{R} \bar{\Upsilon}_{i}
$$

where

$$
\bar{\Upsilon}_{i} \triangleq \int_{0}^{\infty} \frac{1}{\bar{a}_{i} P z_{i}+1} p_{\left|g_{i}\right|^{2}}\left(z_{i}\right) d z_{i}=e^{-\phi_{g_{i}}} \int_{0}^{\infty} \frac{e^{-y_{i}}}{\left(\bar{a}_{i} P \sigma_{g_{i}}^{2} y_{i}+1\right)} I_{0}\left(2 \sqrt{\phi_{g_{i}} y_{i}}\right) d y_{i}
$$


$y_{i} \triangleq z_{i} / \sigma_{g_{i}}^{2}$ and $\phi_{g_{i}} \triangleq\left|\mu_{g_{i}}\right|^{2} / \sigma_{g_{i}}^{2}$. Let us break up the integral (59) into two terms as $\int_{0}^{\infty}=\int_{0}^{1 / P}+\int_{1 / P}^{\infty}$ and use the following results from [36] to approximate $\bar{\Upsilon}_{i}$ :

$$
\begin{array}{r}
\int_{0}^{1 / P} e^{-y_{i}} I_{0}\left(2 \sqrt{\phi_{g_{i}} y_{i}}\right) d y_{i}=\frac{1}{P}+\mathcal{O}\left(P^{-2}\right) \approx \frac{1}{P} \\
\int_{1 / P}^{\infty} y_{i}^{-1} e^{-y_{i}} I_{0}\left(2 \sqrt{\phi_{g_{i}} y_{i}}\right) d y_{i}=E_{1}\left(P^{-1}\right)+\sum_{k=1}^{\infty} \frac{\phi_{g_{i}}^{k}}{k ! k}
\end{array}
$$

where $E_{1}(q) \triangleq-\tilde{c}-\log q-\sum_{k=1}^{\infty} \frac{(-1)^{k} q^{k}}{k ! k}$ for $q>0$, and $\tilde{c}$ denotes the Euler's constant. Note that if $\log P \gg 1$, then $E_{1}\left(P^{-1}\right) \approx \log P$. Using the latter fact, from 59 - 51 we obtain for the case of large $P$ that

$$
\overline{\mathrm{SER}} \leq \frac{c_{1}}{2} \prod_{i=1}^{R} \frac{e^{-\phi_{g_{i}}}}{\bar{a}_{i} \sigma_{g_{i}}^{2}} \frac{\left(\log P+q_{i}\right)}{P}
$$

where $q_{i} \triangleq \bar{a}_{i} \sigma_{g_{i}}^{2}+\sum_{k=1}^{\infty} \frac{\phi_{g_{i}}^{k}}{k ! k}$. Defining

$$
\kappa \triangleq \frac{c_{1}}{2} \prod_{i=1}^{R} \frac{e^{-\phi_{g_{i}}}}{\bar{a}_{i} \sigma_{g_{i}}^{2}}
$$

and using the properties of the logarithm, we can rewrite (62) as

$$
\overline{\mathrm{SER}} \leq \kappa\left(P^{-R\left(1-\frac{\log \log P}{\log P}\right)}+\left(\prod_{i=1}^{R} q_{i}\right) P^{-R}\right) .
$$

For large values of $P$, the first term in the sum in (63) will dominate. Hence, Proposition 1 is proved.

\section{REFERENCES}

[1] E. Larsson and P. Stoica, Space-Time Block Coding for Wireless Communications, Cambridge University Press, Cambridge, 2003.

[2] A. Paulraj, R. Nabar and D. Gore, Introduction to Space-Time Wireless Communications, Cambridge Univ. Press, 2003.

[3] Space-Time Processing for MIMO Communications, A. B. Gershman and N. D. Sidiropoulos (Editors), John Wiley \& Sons, Chichester, UK, 2005.

[4] J. N. Laneman and G. W. Wornell, "Distributed space-time-coded protocols for exploiting cooperative diversity in wireless networks," IEEE Trans. Inform. Theory, vol. 9, pp. 2415-2425, Oct. 2003.

[5] A. Sendonaris, E. Erkip, and B. Aazhang, "User cooperation diversity-Part I: System description," IEEE Trans. Commun., vol. 51, pp. 1927-1938, Nov. 2003.

[6] A. Sendonaris, E. Erkip, and B. Aazhang, "User cooperation diversity-Part II: Implementation aspects and performance analysis,” IEEE Trans. Commun., vol. 51, pp. 1938-1948, Nov. 2003.

[7] M. Gastpar and M. Vetterli, “On the capacity of large Gaussian relay networks," IEEE Trans. Inform. Theory, vol. 51, pp. 765-779, March 2005.

[8] R. U. Nabar, H. Bölcskei, and F. W. Kneubühler, "Fading relay channels: Performance limits and space-time signal design," IEEE J. Select. Areas Commun., vol. 22, pp. 1099-1109, August 2004. 
[9] K. Azarian, H. El Gamal, and P. Schniter, "On the achievable diversity-multiplexing tradeoff in half-duplex cooperative channels," IEEE Trans. Inform. Theory, vol. 51, pp. 4152-4172, Dec. 2005.

[10] A. F. Dana and B. Hassibi, "On the power-efficiency of sensory and ad hoc wireless networks," IEEE Trans. Inform. Theory, vol. 52, pp. 2890-2914, July 2006.

[11] V. Tarokh, N. Seshadri, and A. R. Calderbank, "Space-time codes for high date rate wireless communication: Performance criterion and code construction,” IEEE Trans. Inform. Theory, vol. 44, pp. 744-765, March 1998.

[12] S. Alamouti, "A simple transmit diversity technique for wireless communications," IEEE J. Select. Areas Commun., vol. 16, no. 8, pp. 1451-1458, Oct. 1998.

[13] Y. Jing and B. Hassibi, "Distributed space-time coding in wireless relay networks," IEEE Trans. Wireless Commun., vol. 5, pp. 3524-3536, Dec. 2006.

[14] Y. Jing and H. Jafarkhani, "Using orthogonal and quasi-orthogonal designs in wireless relay networks," IEEE Trans. Inform. Theory, vol. 53, pp. 4106-4118, Nov. 2007.

[15] V. Tarokh, H. Jafarkhani, and A. R. Calderbank, "Space-time block codes from orthogonal designs," IEEE Trans. Inform. Theory, vol. 45, pp. 1456-1467, July 1999.

[16] H. Jafarkhani, "A quasi-orthogonal space-time block code,” IEEE Trans. Commun., vol. 49, pp. 1-4, Jan. 2001.

[17] W. Su and X. Xia, "Signal constellations for quasi-orthogonal space-time block codes with full diversity," IEEE Trans. Inform. Theory, vol. 50, pp. 2331-2347, Oct. 2004.

[18] X.-B. Liang, "Orthogonal designs with maximal rates," IEEE Trans. Inform. Theory, vol. 49, pp. 2468-2503, Oct. 2003.

[19] G. S. Rajan and B. S. Rajan, "Multi-group ML decodable collocated and distributed space-time block codes," IEEE Trans. Inform. Theory, submitted, available as arXiv:0712.2384 2 .

[20] G. S. Rajan and B. S. Rajan, "Distributed space-time codes for cooperative networks with partial CSI," in Proc. IEEE WCNC'07, Hong Kong, March 2007.

[21] P. Larsson, "Large-scale cooperative relaying network with optimal combining under aggregate relay power constraint," in Proc. Future Telecomm. Conf., Bejing, China, 2003, pp. 166-170.

[22] Y. Jing and H. Jafarkhani, "Network beamforming using relays with perfect channel information," IEEE Trans. Inform. Theory, submitted, available as arXiv:0804.1117 1.

[23] Z. Ding, W. H. Chin, and K. K. Leung, "Distributed beamforming and power allocation for cooperative networks," IEEE Trans. Wireless Commun., vol. 7, pp. 1817-1822, May 2008.

[24] V. Havaray-Nassab, S. Shahbazpanahi, A. Grami, and Z.-Q. Luo, "Network beamforming based on second-order statistics of the channel state information," in Proc. ICASSP'08, Las Vegas, USA, Apr. 2008, pp. 2605-2608.

[25] L. Dong, A. P. Petropulu, and H. V. Poor, "A cross-layer approach to collaborative beamforming for wireless ad hoc networks," IEEE Trans. Signal Process., vol. 56, pp. 2981-2993, July 2008.

[26] H. Chen, A. B. Gershman, and S. Shahbazpanahi, "Filter-and-forward distributed beamforming for relay networks in frequency selective fading channels," in Proc. ICASSP'09, Taipei, Taiwan, April 2009, pp. 2269-2272.

[27] E. Koyuncu, Y. Jing, and H. Jafarkhani, "Distributed beamforming in wireless relay networks with quantized feedback," IEEE J. Select. Areas Commun., vol. 26, pp. 1429-1439, Oct. 2008.

[28] J. M. Paredes, B. H. Khalaj, and A. B. Gershman, "Using orthogonal designs with feedback in wireless relay networks," in Proc. IEEE Workshop on Signal Processing Advances for Wireless Commun., Recife, Brazil, July 2008, pp. 61-65.

[29] J. M. Paredes, B. H. Khalaj, and A. B. Gershman, "A differential cooperative transmission scheme with low rate feedback," in Proc. ICASSP'09, Taipei, Taiwan, April 2009, pp. 2621-2624. 
[30] R. W. Heath Jr. and A. J. Paulraj, "A simple scheme for transmit diversity using partial channel feedback," in Proc. Asilomar Conf. on Signals, Systems and Computers, Nov. 1998, pp. 1073-1078.

[31] K. K. Mukkavilli, A. Sabharwal, M. Orchard, and B. Aazhang, "Transmit diversity with channel feedback," in Proc. Int. Symp. on Telecommunications, Tehran, Iran, 2001, pp. 900-901.

[32] J. Akhtar and D. Gesbert, "Extending orthogonal block codes with partial feedback," IEEE Trans. Wireless Commun., vol. 3, pp. 1959-1962, Nov. 2004.

[33] R. Mudumbai, J. Hespanha, U. Madhow, and G. Barriac, "Scalable feedback control for distributed beamforming in sensor networks," in Proc. ISIT'05, Adelaide, Australia, Sept. 2005, pp. 137-141.

[34] R. Mudumbai, J. Hespanha, U. Madhow and G. Barriac, "Distributed transmit beamforming using feedback control," IEEE Trans. Inform. Theory, submitted, available as arXiv:cs/0603072 v1 [cs.IT].

[35] M. Johnson, M. Mitzenmacher, and K. Ramchandran, “Distributed beamforming with binary signaling,” in Proc. ISIT'08, Toronto, ON, July 2008, pp. 890-894.

[36] Y. Jing and H. Jafarkhani, “Network beamforming with channel mean and covariance at relays," in Proc. ICC'08, Beijing, May 2008, pp. 3743-3747.

[37] W. K. Ma, T. N. Davidson, K. M. Wong, Z.-Q. Luo, and P.-C. Ching, "Quasi-maximum-likelihood multiuser detection using semi-definite relaxation with application to synchronous CDMA,” IEEE Trans. Signal Processing, vol. 50, pp. 912-922, April 2002.

[38] S. Boyd and L. Vandenberghe, Convex Optimization, Cambridge University Press, 2004.

[39] N. D. Sidiropoulos, T. N. Davidson, and Z.-Q. Luo, “Transmit beamforming for physical layer multicasting," IEEE Trans. Signal Processing, vol. 54, pp. 2239-2251, June 2006.

[40] Y. Jing and H. Jafarkhani, "Distributed differential space-time coding for wireless relay networks," IEEE Trans. Commun., vol. 56, pp. 1092-1100, July 2008.

[41] M. K. Simon and M.-S. Alouni, Digital Communication Over Fading Channels, John Wiley \& Sons, Second Edition, 2005.

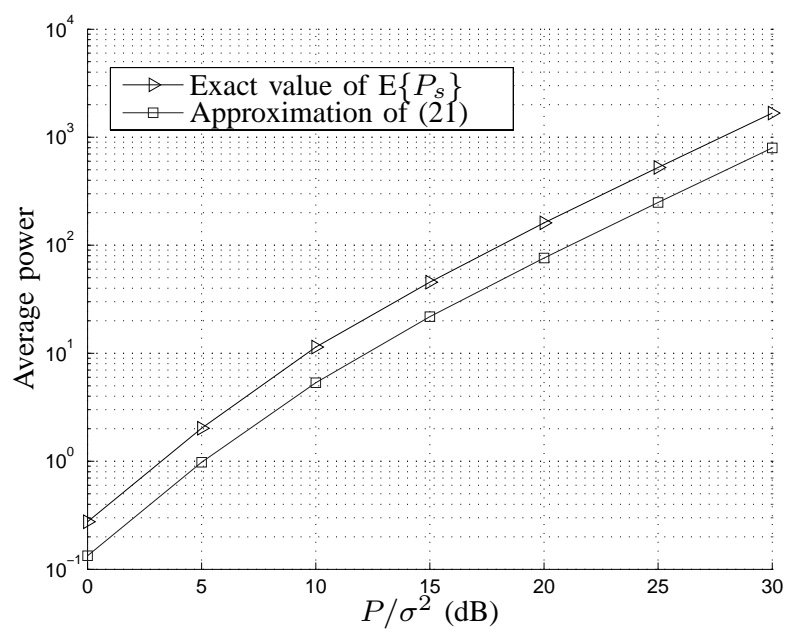

Fig. 1. Comparison of the approximation and the exact value of $\mathrm{E}\left\{P_{s}\right\}$. 


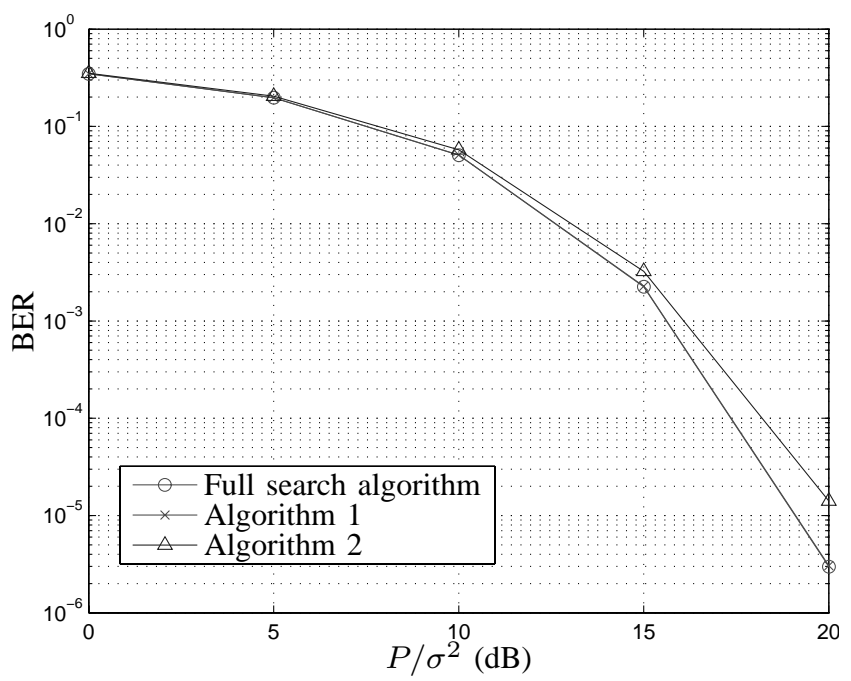

Fig. 2. BERs versus $P / \sigma^{2}$; first example.

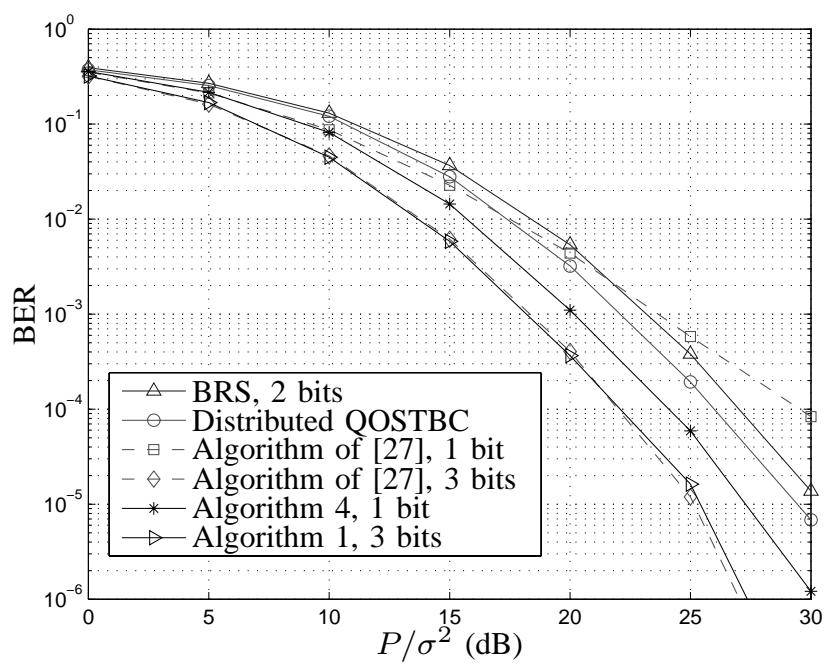

Fig. 3. BERs versus $P / \sigma^{2}$; second example. 


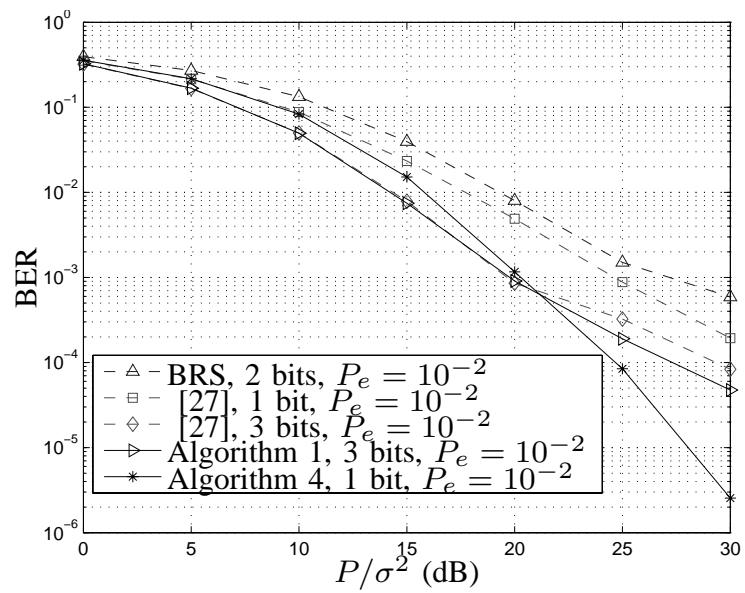

Fig. 4. BERs versus $P / \sigma^{2}$; second example.

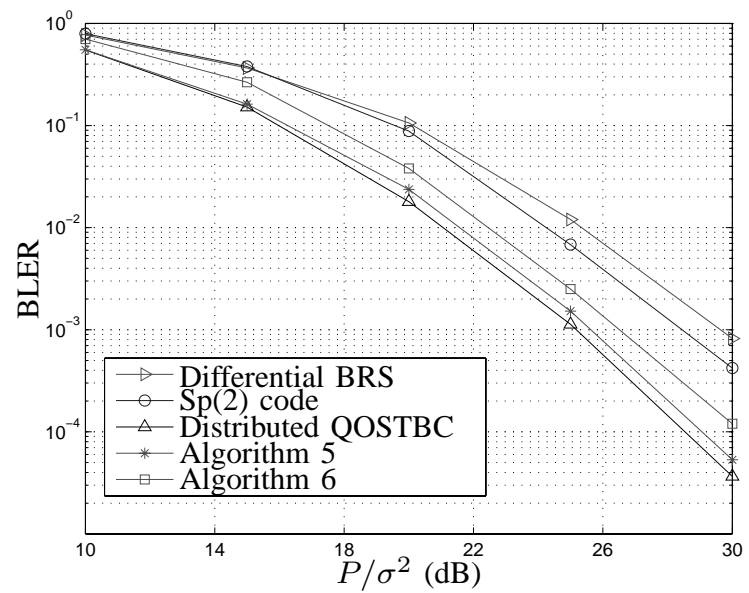

Fig. 5. BLER versus $P / \sigma^{2}$; third example.

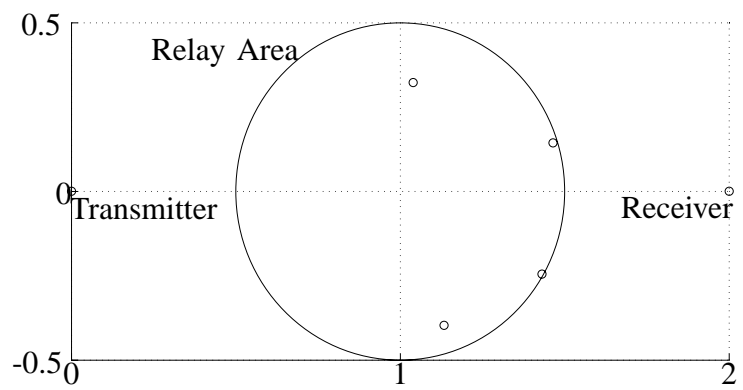

Fig. 6. Geometry of the fourth example. 


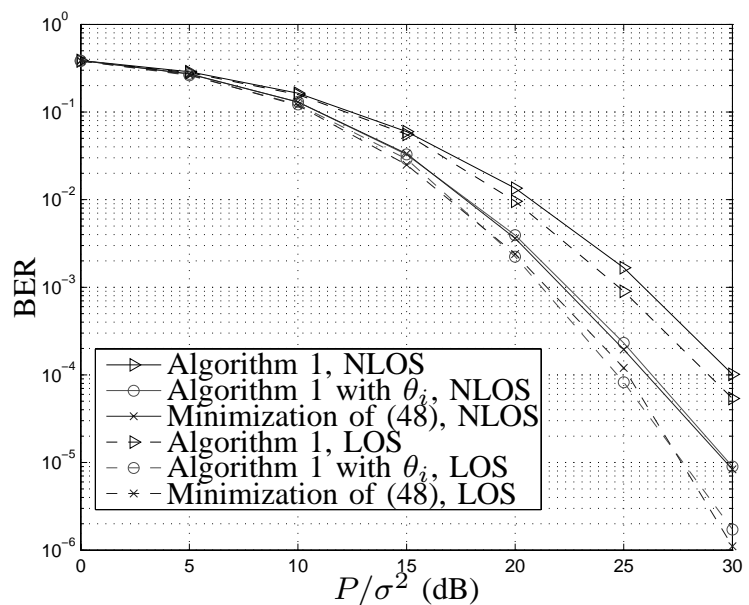

Fig. 7. BER versus $P / \sigma^{2}$; fourth example.

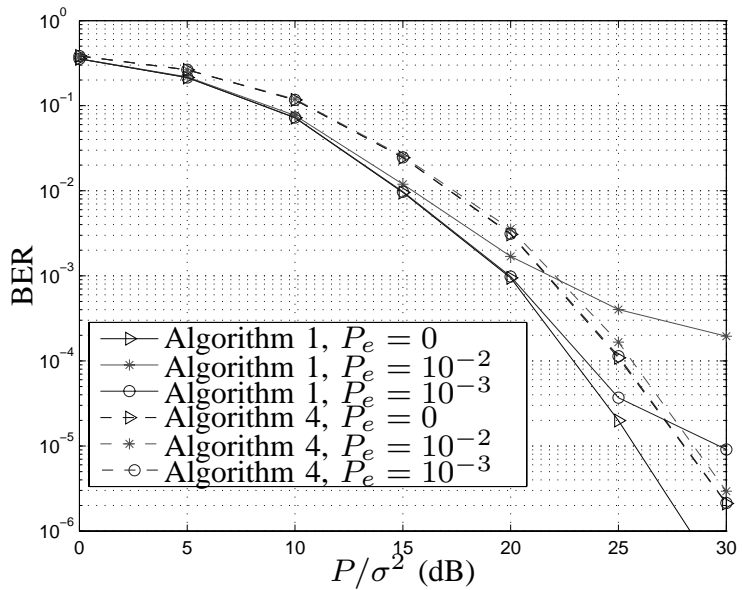

Fig. 8. BER versus $P / \sigma^{2}$; fifth example. 